\title{
Serial cerebral CT abnormalities in relapsing acute disseminated encephalomyelitis
}

\author{
R W H WALKER, J GAWLER \\ From the Department of Neurological Sciences, St Bartholomew's Hospital, London, UK
}

SUMMARY A 7 year old girl developed acute disseminated encephalomyelitis following a $M y c o-$ plasma pneumoniae respiratory infection. The illness followed a relapsing course during the first two months. Computed tomography (CT) showed cerebral lesions of a severity and extent out of proportion to the clinical manifestations. The CT abnormalities altered with changes in her clinical state.

Acute disseminated encephalomyelitis (ADEM) is thought to be an autoimmune mediated disorder which is usually post-infectious or follows immunisation. ${ }^{1-3}$ The pathology affects white matter principally, with perivenous lymphocytic infiltration and foci of demyelination which may become confluent. Relapsing cases have been described. ${ }^{148}$ The relationships between ADEM, relapsing ADEM and multiple sclerosis are a matter of speculation. ${ }^{19}$ Clinically, ADEM may present in a manner identical to acute viral encephalitis, but in some cases of ADEM focal neurological deficits are the predominating clinical manifestations. ${ }^{10}$

We report a girl with relapsing ADEM in whom the cerebral computed tomography (CT) appearances were alarmingly severe even at times when clinical manifestations were relatively slight.

\section{Case report}

A 7 year old right-handed girl, born in the UK to parents of Italian origin, was admitted on 19 December 1986. Two weeks prior to admission she had developed a moderately severe productive cough lasting several days, and for one week had suffered progressive weakness of the right leg without any other symptoms. There was no past medical or family history.

Examination revealed right upper quadrant visual inatten-

Address for reprint requests: Dr J Gawler, Department of Neurological Sciences, St Bartholomew's Hospital, West Smithfield, London EC1A 7BE, UK

Received 13 December 1988.

Accepted 22 March 1989 tion and a spastic monoparesis of the right leg with weakness maximal in the foot. The right plantar response was extensor. There were no other signs. Cerebral CT showed three large discrete areas of low attenuation, in the subcortical white matter of the right frontal, left occipito-parietal and left fronto-parietal regions. The last lesion extended to involve cortical grey matter. There was a striking absence of mass effect. Each lesion showed ring enhancement after contrast administration (fig 19.12.86).

EEG was abnormal with focal left parietal and right frontal slow waves. Relevant negative or normal tests were: chest radiograph, full blood count, erythrocyte sedimentation rate, antinuclear antibodies, cold agglutinins, serum complement $\left(C^{\prime} 3\right)$, viral (including HIV) and toxoplasma antibodies, and blood cultures. Mycoplasma pneumoniae complement fixation test results were positive (titre 512 both on the day of admission and 8 days later). IgM antibodies to $M$ pneumoniae were detected.

Prednisolone, $30 \mathrm{mg}$ daily $(1.2 \mathrm{mg} / \mathrm{kg})$, was started on 20 December, but was tailed off after 10 days (discontinued on 15 January 1987) as there had been little clinical response, although a second CT scan on 31 December showed partial resolution of the lesions, and an absence of contrast enhancement (fig 31.12.86). On 20 January 1987 the family noticed that the patient was not using her right hand, and this was followed by progressive weakness of the limb leading to readmission two days later. On examination the visual field defect had resolved and power in her right leg had improved but there was now right upper limb weakness of pyramidal distribution, maximal in the hand. Her third CT scan (fig 23.1.87) showed a new right posterior frontal ring enhancing lesion and confluence of the left hemisphere lesions with recurrence of ring enhancement. Cerebrospinal fluid (CSF) contained 10 mononuclear cells $/ \mathrm{mm}^{3}$, with normal total protein, IgG and glucose concentrations. CSF protein electrophoresis was normal. Visual evoked potentials (VEP) to a checkerboard pattern reversal stimulus were normal. 

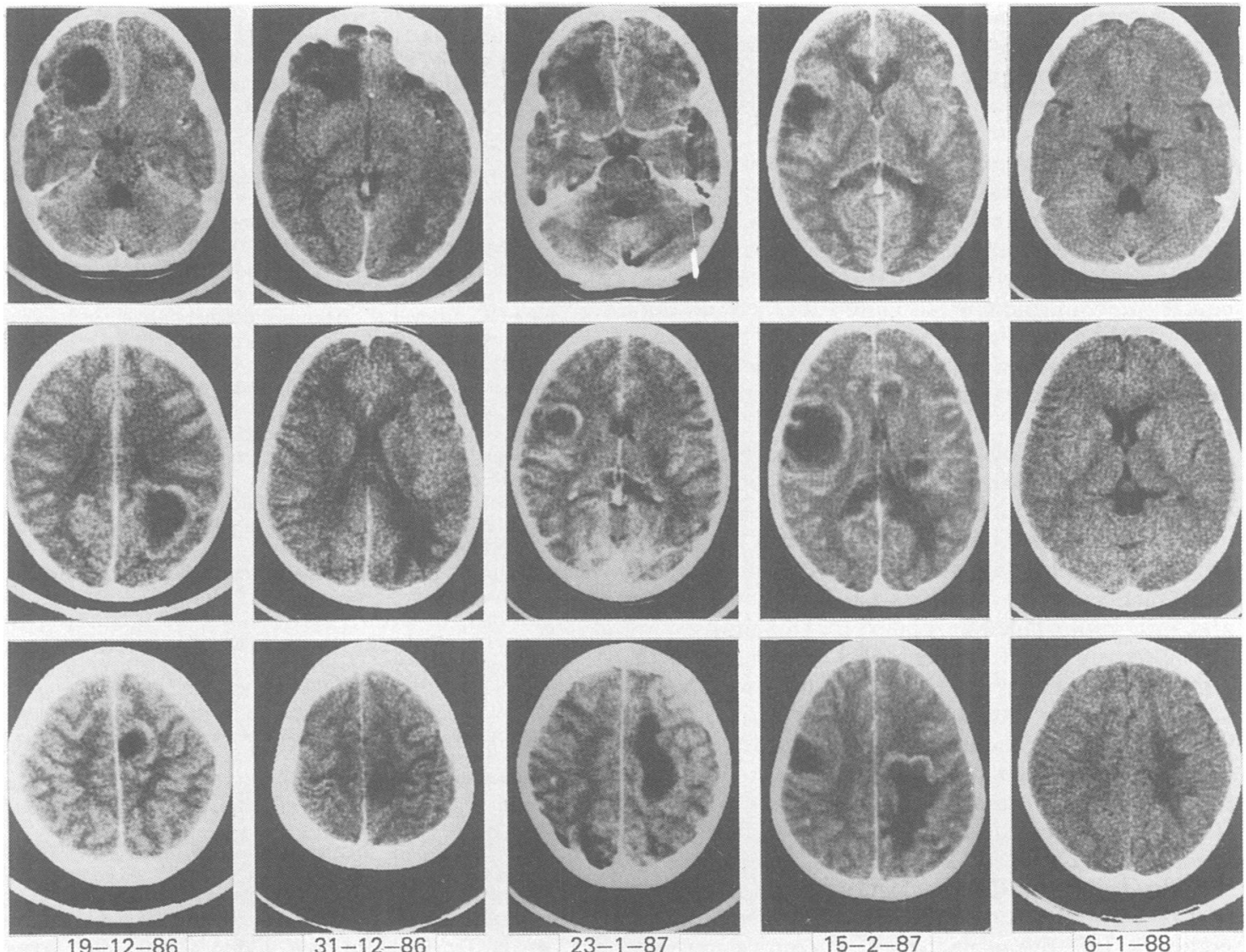

Fig Serial cerebral CT examination, on dates shown, all following contrast except 6.1.88. The levels of the cuts illustrated vary throughout the series to show the maximum extent of the lesions.

A 15 day course of erythromycin was administered, even though $M$ pneumoniae had not been cultured at any stage. Considerable clinical improvement was noted during her admission. However, shortly after discharge on 9 February 1987 , she became reluctant to speak or eat and dribbling was noted. On re-admission on 13 February she was dysarthric with bilateral weakness of facial, palatal and tongue muscles. The power of her right hand had deteriorated and tone was now increased in both legs.

The fourth CT scan (fig 15.2.87) showed expansion of the right posterior frontal lesion, a persisting extensive enhancing subcortical left occipito-parietal lesion, and bilateral frontal periventricular low attenuation areas.

Prednisolone $30 \mathrm{mg}$ daily was restarted, this time with prompt and dramatic improvement in her condition. During the remainder of 1987 she remained well taking alternate day prednisolone in reducing doses. Functional disability had resolved by the autumn of 1987 .

A follow-up CT scan was obtained in January 1988, without contrast (which she refused). It showed resolution of all lesions save for a small area of lucency in the white matter of the left hemisphere (fig 6.1.88).

Steroids were withdrawn in May 1988. She remains asymptomatic. Slowness of repetitive movement of the right hand and slight right-sided hyperreflexia are the only residual signs.

\section{Discussion}

There was a clear history of an antecedent respiratory tract infection with serological evidence of recent Mycoplasma pneumoniae infection. " A wide range of inflammatory processes may affect the nervous system after $M$ pneumoniae infection, including meningitis, encephalitis, transverse myelitis, cranial mononeuropathy and Guillain-Barré syndrome. ${ }^{1213}$ Only very rarely has $M$. pneumonia been isolated from spinal fluid ${ }^{14}$ and it is likely that the majority reflect post-infectious autoimmune processes. ${ }^{15} 16$

In this case the diagnosis of ADEM was made at 
first presentation on clinical grounds, and the CT appearances were interpreted as indicating a mainly white matter inflammatory encephalopathy rather than multiple abscesses or multifocal neoplasia. Most cases of ADEM are steroid-responsive..$^{17}$ In our patient the first course of prednisolone was followed by little clinical improvement, although there was loss of contrast enhancement of CT lesions. There were two subsequent relapses, with new clinical and CT abnormalities. It is possible that the effect of the first course of prednisolone was to interrupt the evolution of the ADEM, creating an apparent rather than a real relapse. Even if this were the case it nevertheless leaves one relapse which was not related to steroid administration.

Although relapsing ADEM is well reported, ${ }^{148}$ it is uncommon. The relapsing and remitting course led us to consider a diagnosis of multiple sclerosis, as this disease is seen in young children, albeit rarely. ${ }^{1819}$ However, the CT abnormalities were much more florid than is usual in multiple sclerosis ${ }^{2021}$ and the CSF and VEP results failed to support that diagnosis.

The cerebral CT appearances associated with ADEM have been reported, ${ }^{1022}$ and include areas of white matter low attenuation with or without peripheral contrast enhancement, thought to indicate demyelination. Brain swelling may be seen. However, $\mathrm{CT}$ is frequently normal. Magnetic resonance imaging shows extensive multifocal white matter abnormalities in this condition. ${ }^{2324}$

In this patient the CT abnormalities were so marked that cerebral biopsy was seriously considered, lest a multifocal infective or neoplastic process was missed. Clearly biopsy in patients with such a favourable outcome is undesirable unless absolutely necessary, and thus it is important to appreciate the severity of CT abnormality which may be encountered in ADEM.

\section{References}

1 Alvord EC. Disseminated encephalitis: its variations in form and their relationships to other diseases of the nervous system. In: Koetsier JC, ed. Handbook of Clinical Neurology. Vol 3(47): Demyelinating Diseases. Amsterdam, North Holland, 1985:467-502.

2 Johnson RT. The pathogenesis of acute viral encephalitis and postinfectious encephalomyelitis. $J$ Infect Dis 1987;155: $359-64$.
3 Hart MN, Earle KM. Haemorrhagic and perivenous encephalitis: a clinical-pathological review of 38 cases. J Neurol Neurosurg Psychiatry 1975;38:585-91.

4 Alcock NS, Hoffman HL. Recurrent encephalomyelitis in childhood. Arch Dis Child 1962;37:40-4.

5 Durston JHJ, Milnes JN. Relapsing encephalomyelitis. Brain 1970;93:715-30.

6 Yahr MD, Lobo-Antunes J. Relapsing encephalomyelitis following the use of influenza vaccine. Arch Neurol 1972;27:182-3.

7 Poser CM, Roman G, Emery S. Recurrent disseminated vasculomyelinopathy. Arch Neurol 1978;35:166-70.

8 Behan PO, Henderson LM, Morrison L. Recurrent acute disseminated encephalomyelitis. J Neuroimmunol 1987;16:16.

9 Miller HG, Evans MJ. Prognosis in acute disseminated encephalomyelitis; with a note on neuromyelitis optica. $Q \mathrm{~J}$ Med 1953;22:347-79.

10 Pasternak, JF, De Vivo DC, Prensky AL. Steroid-responsive encephalomyelitis in childhood. Neurology 1980;30:481-6.

11 Smith TF. Mycoplasma pneumonia infections: diagnosis based on immunofluorescence titer of IgG and IgM antibodies. Mayo Clin. Proc. 1986;61:830-1.

12 Lerer RJ, Kavalsky SM. Central nervous system disease associated with mycoplasma pneumonia infection. Pediatrics 1973;52:658-68.

13 Broughton RA. Infections due to mycoplasma pneumonia in childhood. Pediatr Infect Dis 1986;5:71-85.

14 Kasahara I, Otsubo Y, Yanase T, Oshima H, Ichimaru H, Nakamura M. Isolation and characterisation of mycoplasma pneumoniae from a patient with pneumonia and meningoencephalitis. J Infect Dis 1985;152:823-5.

15 Pönkä A. Central nervous system manifestations associated with serologically verified mycoplasma pneumoniae infection. Scand J Infect Dis 1980;12:175-84.

16 Behan PO, Feldman RG, Segarra JM, Draper IT. Neurological aspects of mycoplasmal infection. Acta Neurol Scand 1986;74:314-22.

17 Ziegler DK. Acute disseminated encephalitis. Arch Neurol 1966;14:476-88.

18 Hauser SL, Bresnan MJ, Reinherz EL, Weiner HL. Childhood multiple sclerosis: clinical features and demonstration of changes in $\mathrm{T}$ cell subsets with disease activity. Ann Neurol 1982;11:463-8.

19 Duquette P, Murray TJ, Pleines J, Ebers GC, et al. Multiple sclerosis in childhood: clinical profile in 125 patients. J Paediatr 1987;111:359-63.

20 Cala LA, Mastaglia FL, Black JL. Computerised tomography of brain and optic nerve in multiple sclerosis. J Neurol Sci 1978;36:411-26.

21 Lane B, Carroll BA, Pedley TA. Computerised cranial tomography in cerebral disease of white matter. Neurology 1978;28: 534-44.

22 Lukes SA, Norman D. Computerised tomography in acute disseminated encephalomyelitis. Ann Neurol 1983;13:567-72.

23 Atlas SW, Grossman RI, Goldberg HI, Hackney DB, Bilaniuk LT, Zimmerman RA. MR diagnosis of acute disseminated encephalomyelitis. J Comput Assist Tomogr 1986;10:798-801.

24 Dunn V, Bale JF, Zimmerman RA, Perdue Z, Bell WE. MRI in children with postinfectious disseminated encephalomyelitis. Magnetic Resonance Imaging 1986;4:25-32. 